\title{
SPECIAL COMMUNICATION \\ LONG-TERM AMBULATORY ECG MONITORING AN ESSENTIAL DIAGNOSTIC TOOL FOR CURRENT TIMES
}

\author{
Pir Sheeraz Ali ${ }^{1}$, Syed Haseeb Raza ${ }^{2}$, Sarah Mansoor $^{3}$ \\ ${ }^{1}$ National Institute of Cardiovascular Diseases, Karachi, Pakistan, ${ }^{2}$ Chaudhary Pervaiz Elahi Institute of Cardiology, Multan, Pakistan, \\ ${ }^{3}$ Aga Khan University Hospital, Karachi, Pakistan
}

Heart valve problems affect more than 100 million people worldwide. According to statistics, around $55 \%$ of valvular diseases are treated by a mechanical prosthesis. The first heart valve replaced model was the caged-ball valve, more than 50 models of heart valves designed by different companies. Each design has different aspects such as valve geometry, leaflets design, materials used for model manufacturing, coating techniques, and coating materials. Depending on the patient's need and condition, the native heart valve either replaced by a biological or mechanical heart valve. Biological valves are made of living tissues whereas mechanical valves manufactured by the biomaterials, which are biocompatible and do not causes any reaction inside the body. The prototype discussed in this paper provides good hemocompatibility, because of the biomaterial used in this prototype manufacturing. It will reduce tissue ingrowth, due to the enhanced leaflet ear of the orifice ring. Moreover, it will cause less thrombotic ef fects into the host due to greater contact angel of graphite and smooth surface of graphite after pyrolytic coating. The significant evolution of mechanical valve designs consists of valve geometry, coating technique, and materials. In this research, the 3D-CAD model of Bileaflet Mechanical Mitral Heart Valve was designed using SOLID WORKS 2016 and fabricated by 5 axis Computer Numeric Control (CNC) machine. Graphite was used for the fabrication of prototype and Pyrolytic Carbon (PyC) coating was performed with Chemical Vapor deposition (CVD) technique. Scanning electron microscopy (SEM), Fourier Transform Infrared Spectroscopy (FTIR), and X-ray Diffraction (XRD) were used to determine the effects of CVD on surface topography and chemical structure of graphite model before and after coating. Furthermore, hemocompatibility of graphite and $\mathrm{PyC}$ analyzed through in-vitro hemolytic activity. The Characterization results showed that the Bileaflet Mechanical Mitral Heart valve prototype after $\mathrm{PyC}$ coating provides a smooth surface with improved hemocompatibility and less adhesion. Besides, the Mechanical Heart valves showed no hemolysis during the hemolytic activity. By virtue of its smooth and nonporous surface, it is antithrombotic and provides good hemodynamics. The advance long leaflet ear design reduces the tissue ingrowth around the orifice which will further limit the leaflets movement.

Keywords: Mechanical Heart valve, Biocompatibility, Biomaterials, Hemodynamics, Pyrolytic carbon Coating

Citation: Ali PS, Raza SH, Mansoor S. Long-Term Ambulatory ECG Monitoring an Essential Diagnostic Tool for Current Times. Pak Heart J. 2021;54(04):380-382. DOI: https://doi.org/10.47144/phj.v54i4.2117

\section{INTRODUCTION}

Ambulatory ECG (AECG) monitoring with diary correlation of symptoms has been proven to provide significant diagnostic, therapeutic and prognostic benefit with an arrhythmic cause of symptoms. Arrhythmias can range from premature atrial and ventricular complexes (APCs/ PVCs usually benign), to Atrial and Ventricular Fibrillation which causes significant morbidity and mortality. Symptoms such as palpitations, shortness of breath, chest pain and syncope are common during arrhythmias and their frequency determines the choice of investigation needed to diagnose the arrythmia.

Arrhythmias can be a manifestation of many cardiac and non-cardiac diseases. These also include congenital diseases and are often missed due to inadequate monitoring. Since most arrhythmias are intermittent they are more likely to be detected during extended ECG monitoring. Other uses of ambulatory ECG devices include ST segment analysis, heart rate variability, signal averaged ECGs, diurnal QT and QTc analysis (including patients with long QT) ${ }^{1}$ obstructive sleep apnea and vectorcardiography. ${ }^{2}$ These factors have been shown to have relation to significant cardiovascular diseases aiding the diagnosis of various arrhythmias.

Syncope although mostly benign, could potentially be a consequence of a life-threatening arrhythmia in up to $20 \%$ patients. $^{3}$ Nonetheless syncope poses a significant cause of disturbance in a patients' life and 
definitive diagnosis is necessary to ensure patients well-being.

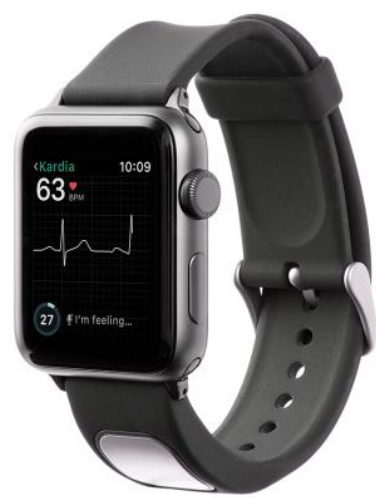

\section{Figure 1: FDA Approved Apple Smart Watch}

ESC Guidelines on Syncope (2018) recommend further testing with AECG via Holter monitoring, wearable patch recorder, external and internal loop recorders etc. depending on the frequency after initial examination is negative for a definite cause.

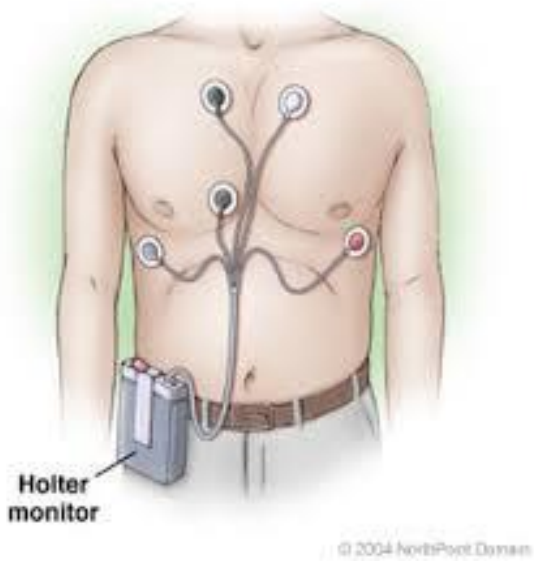

Figure 2: Holter Monitor

Atrial Fibrillation (AF) increases risk of stroke more than five times. Atrial Fibrillation diagnosed after stroke is an important hallmark of recurrent stroke risk. Many studies have demonstrated post stroke AECG increases the chances of detecting $\mathrm{AF}(15 \%$ vs. $5 \%$ when compared to standard monitoring. An increase incidence in atrial arrhythmias (atrial high rate episodes AHRE) has been seen in patients with Permanent Pacemakers which should be documented by AECG to be treated accordingly. ${ }^{4}$ Uses can be prognostic if rate was to be monitored in $\mathrm{AF}$ to assess efficacy of rate control treatment and offer adequate anticoagulation according to the 2020 ESC atrial fibrillation guidelines.
Some limitations of twenty-four hours Holter monitoring have recently been overcome by improvements in hardware and software technology including adhesive patches and wireless telemetry. Newer adhesive patches are softer, waterproof and electrode free monitors which offer unprecedented mobility and ease of carrying out daily routine by the patient. They operate as either recorders or wireless streaming devices. ${ }^{5}$ These devices were safe and effective during the pandemic even when delivered home through mail to critically ill patients. ${ }^{6}$

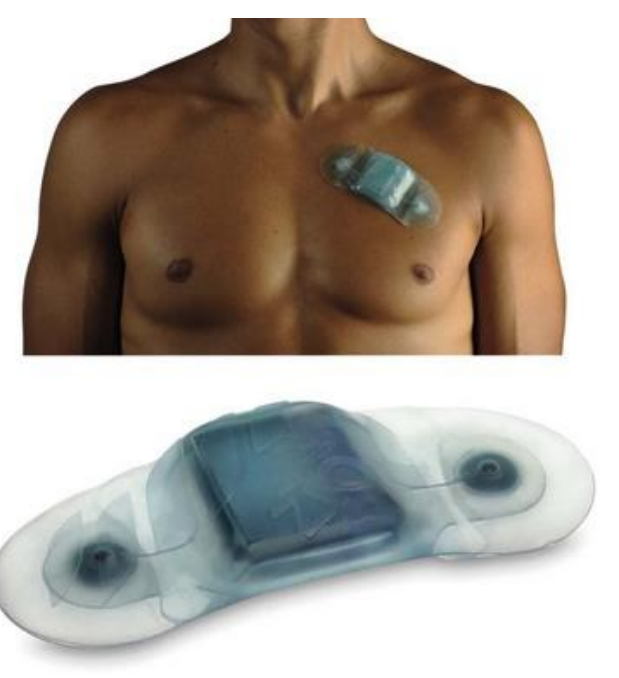

Figure 4: Implantable Cardiac Monitor

The advent of smart phones has added endless potential for recording through wireless Bluetooth transmission. Smart devices like the OMSHIRTtm have the added advantage of being comfortable to wear. Newer devices for example Cardiostat has been shown to offer equal quality tracings when compared to standard Holter monitoring, often up to the $99 \%$ sensitivity and specificity through better designed $\mathrm{R}$ wave (QRS) detection algorithms., ${ }^{7,8}$ Studies have shown these newer devices to be easily operable and can even be mailed to patients homes for selfattachment with an equal efficacy to hospital applied machine. ${ }^{6}$

Many studies have shown a preference over intra cardiac monitors (ICM) due to these above mentioned advantages. ${ }^{9}$ The effectiveness of even longer recordings through Implantable Loop Recorder has also been satisfactory when following patients after Ablation therapy leading to practice updating guideline changes in rhythm management. ${ }^{10}$

A recent review article summarized that although physicians in the US had knowledge of how and when to offer monitoring devices based on the frequency of symptoms, they were often seen prescribing Holter monitoring due to familiarity. Data also showed that in 
case the initial investigation was inconclusive, the physician would still repeat the same investigation. ${ }^{3}$

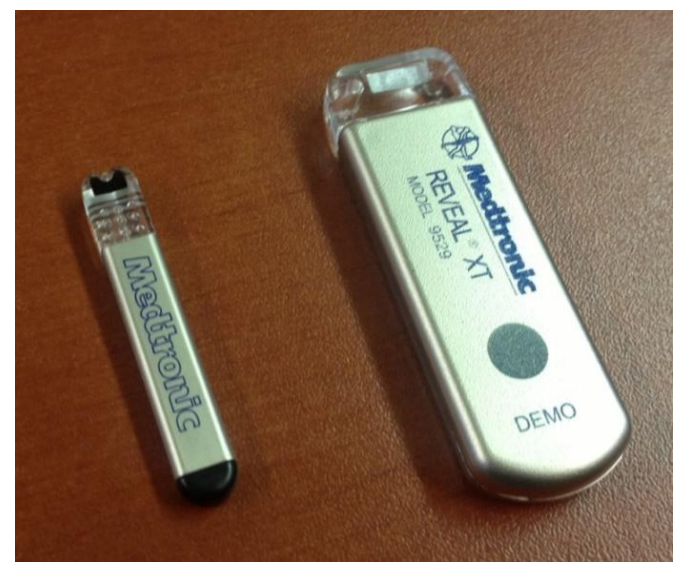

Figure 4: Implantable Cardiac Monitor

In a country like Pakistan where there are limited resources, diagnosis and management of arrhythmias still has a long way to go. This article sheds light on the need of utilizing the recommended available devices.

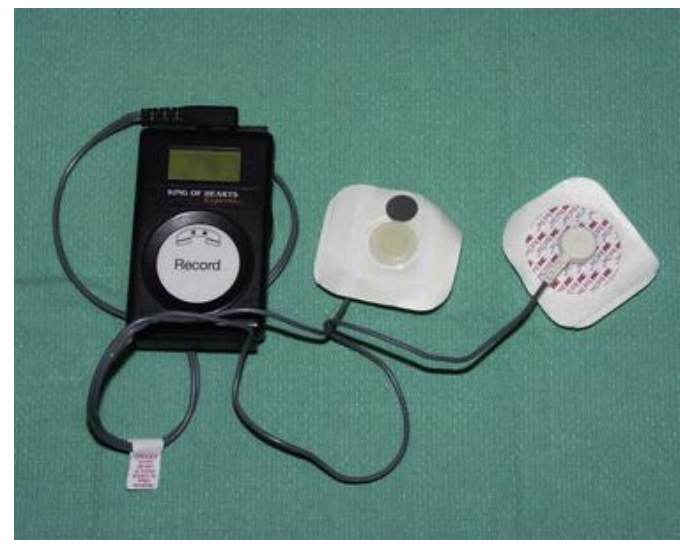

Figure 5: External Loop Recorder

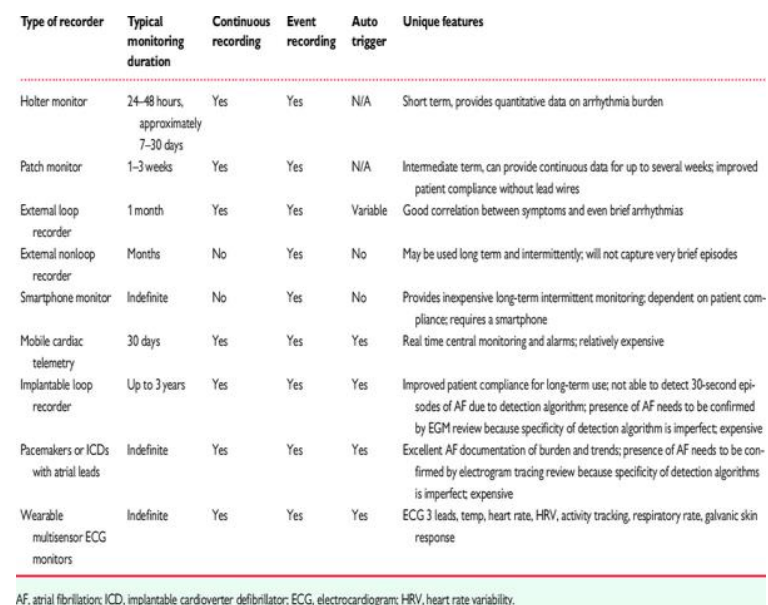

Table 1: Types of Ambulatory ECG Monitoring. ${ }^{11}$

\section{AUTHORS' CONTRIBUTION}

PSA: Concept and design, data acquisition, interpretation, drafting, final approval, and agree to be accountable for all aspects of the work. SHR, SM: Data acquisition, interpretation, drafting, final approval and agree to be accountable for all aspects of the work.

Conflict of interest: Authors declared no conflict of interest.

\section{REFERENCES}

1. Makarov Leonid, Komoliatova Vera, Zakliazmenskaya Elena, Dmitrieva Alena. Abstract 12768: Ambulatory ECG Monitoring in Patients With Long QT Syndrome. Circulation. 2018;138(Suppl_1):A12768-A12768.

2. 2. Mond HG. The Spectrum of Ambulatory Electrocardiographic Monitoring. Heart Lung Circ. 2017;26(11):1160-74.

3. Benditt DG, Adkisson WO, Sutton R, Mears RK, Sakaguchi S. Ambulatory diagnostic ECG monitoring for syncope and collapse: An assessment of clinical practice in the United States. Pacing Clin Electrophysiol. 2018;41(2):203-9.

4. ESC Guidelines on Atrial Fibrillation (Management of) [Internet]. [cited 2021 Apr 22]. Available from: https://www.escardio.org/Guidelines/Clinical-PracticeGuidelines/Atrial-Fibrillation-Management, https://www.escardio.org/Guidelines/Clinical-PracticeGuidelines/Atrial-Fibrillation-Management

5. Lobodzinski SS, Laks MM. New devices for very long-term ECG monitoring. Cardiol J. 2012;19(2):210-4.

6. Benjamin H, Bischof M, Goldshtein D, Fecteau P, Newman D. A pandemic response to home delivery for ambulatory ECG monitoring: Development and validation. J Electrocardiol. 2021;64:72-5.

7. Nault I, André P, Plourde B, Leclerc F, Sarrazin J-F, Philippon F, et al. Validation of a novel single lead ambulatory ECG monitor CardiostatTM - Compared to a standard ECG Holter monitoring. J Electrocardiol. 2019;53:57-63.

8. Melgarejo-Meseguer F-M, Everss-Villalba E, Gimeno-Blanes FJ, Blanco-Velasco M, Molins-Bordallo Z, Flores-Yepes J-A, et al. On the Beat Detection Performance in Long-Term ECG Monitoring Scenarios. Sensors [Internet]. 2018 May 1 [cited 2021 Apr 21];18(5). Available from: https://www.ncbi.nlm.nih.gov/pmc/articles/PMC5982228/

9. Use of Ambulatory Electrocardiographic (Holter) Monitoring | Annals of Internal Medicine [Internet]. [cited 2021 Apr 21]. Available from: https://www.acpjournals.org/doi/10.7326/00034819-113-1-53

10. Davtyan K, Shatakhtsyan V, Poghosyan H, Deev A, Tarasov A, Kharlap M, et al. Radiofrequency versus Cryoballoon Ablation of Atrial Fibrillation: An Evaluation Using ECG, Holter Monitoring, and Implantable Loop Recorders to Monitor Absolute and Clinical Effectiveness. BioMed Res Int. 2018;2018:e3629384.

11. Calkins H, Hindricks G, Cappato R, Kim Y-H, Saad E, Aguinaga L, et al. 2017 HRS/EHRA/ECAS/APHRS/SOLAECE expert consensus statement on catheter and surgical ablation of atrial fibrillation: Executive summary. Ep Europace. 2018;20(1):e1-60.

\section{Address for Correspondence:}

Dr. Pir Sheeraz Ali, National Institute of Cardiovascular Diseases (NICVD), Rafiqui (H.J.) Shaheed Road, Karachi- 75510, Pakistan.

Email: pirsheerazali@gmail.com 